\title{
X-ray Scattering from Self-Assembled InAs Islands
}

\author{
A. Malachias*, B. R. A. Neves*, W. N. Rodrigues*, M. V. B. Moreira*, S. Kycia ${ }^{\dagger}$, \\ T. H. Metzger\#, and R. Magalhães-Paniago ${ }^{* \dagger}$ \\ * Departamento de Física, Universidade Federal de Minas Gerais, Instituto de Ciências Exatas \\ Campus da Pampulha, Caixa Postal 702, 30123-970 Belo Horizonte, Minas Gerais, Brazil \\ \# European Synchrotron Radiation Facility, BP 220, F-38043 Grenoble Cedex, France \\ † Laboratório Nacional de Luz Síncrotron, Caixa Postal 6192, 13084-971, Campinas, São Paulo, Brazil
}

Received on 31 March, 2003

\begin{abstract}
In this work several structural and chemical properties of self-assembled InAs islands grown on $\operatorname{GaAs}(001)$ are studied using surface $\mathrm{x}$-ray scattering with synchrotron radiation. The technique of $\mathrm{x}$-ray diffraction under grazing incidence condition was employed to differentiate coherent and incoherent islands. We used a model of a strained pyramidal island to interpret the $\mathrm{x}$-ray results and correlate size and strain-state of these islands. The degree of GaAs interdiffusion in the islands was inferred from the variation of volume of the unit cell. The Poisson's ratio of the two materials involved establishes a limit of tetragonal distortion for this material. Any variation in this distortion is associated with the presence of Ga inside the islands.
\end{abstract}

\section{Introduction}

The electronic properties of self-assembled islands, such as InAs grown on $\mathrm{GaAs}(001)$ are extremely dependent on their inner strain and chemical state. Although a large number of variables rules island formation only a few features of their final state determines their technological applicability: a) absence of defects like dislocations or stacking faults, directly related to quantum efficiency of quantum dots (QDs); b) morphological homogeneity, which gives optical and electronic response quality and; c) elastic strain and intermixing. Here we employ x-ray scattering to study features (a) and (c), described in two different parts of this work. Firstly we show how x-ray scattering provides accurate quantitative information about the coexistence of coherent and incoherent InAs islands grown in $\mathrm{GaAs}(001)$. Secondly a reciprocal space map enables to observe the threedimensional strain status and infer the chemical composition of InAs nanostructures.

\section{InAs coherent-incoherent transi- tion}

Growing dislocation-free nanostructures with a narrow size distribution is crucial for the optimal performance of optoeletronics quantum-dot devices. Since early work in this field, the transition of InAs dots from coherent to incoherent states has been mostly investigated by Atomic Force Microscopy (AFM) [1]. Samples with coherent and incoherent quantum dots usually exhibit a bimodal size distribution, where coherent (strained) islands are smaller than incoherent (relaxed) nanostructures [1]. However, the exact point of the coherence-incoherence transition is hard to be identified by AFM, since at this point, islands of both types may have nearly the same size. X-ray scattering is used in this paper to separate coherent and incoherent islands by relating their size to strain-state.

The heteroepitaxial growth of InAs on GaAs(001) usually proceeds as follows [1]: while the amount of deposited InAs is less than $\sim 1.6$ monolayers (MLs), a 2-dimensional layer can be grown. Increasing this coverage beyond 1.6 MLs up to $\sim 2.3 \mathrm{MLs}$, coherently strained (dislocation-free) dots are formed. For higher coverages, dislocations start to appear, generating relaxed islands on the surface [1].

For this work, three InAs/GaAs samples were grown by molecular beam epitaxy on a $\operatorname{GaAs}(001)$ substrate. In sample A, 1.9 MLs of InAs were deposited on a GaAs buffer layer at $500{ }^{\circ} \mathrm{C}$ at a rate of $0.16 \mathrm{ML} / \mathrm{s}$ and $\mathrm{As}_{4}$ pressure of $5.8 \times 10^{-5}$ Torr. In sample B, these same steps were adopted and, subsequently, a 30ML GaAs spacer was deposited on the InAs dots, followed by 1.9 MLs of InAs, forming an InAs/GaAs/InAs bilayer. In sample C 3.0 MLs of InAs islands were deposited at $535^{\circ} \mathrm{C}$. AFM measurements confirmed the formation of self-assembled dots.

The X-ray experiments were performed at the European Synchrotron Radiation Facility - ESRF (France) and at the Laboratório Nacional de Luz Síncrotron - LNLS (Brazil). Samples A and B were investigated at TROIKA II - ID10b (ESRF), while the results on sample $\mathrm{C}$ were obtained at the XD1 bending magnet beamline at LNLS. We have used nearly identical experimental setups in both facilities. The XD1 experimental configuration can be seen in fig. 1(a). All measurements were done at grazing incidence geometry (as a function of $\omega$ - sample rotation angle and $2 \theta$ scattering angle). The incident angle $\alpha_{i}$ was set by an in- 
dependent circle and the exit angle $\alpha_{f}$ was measured from $0^{\circ}$ to $1^{\circ}$ by a position sensitive detector (PSD). Samples A and $\mathrm{B}$ (sample $\mathrm{C}$ ) were (was) illuminated at a grazing angle $\alpha_{i}=0.25^{\circ}\left(\alpha_{i}=0.3^{\circ}\right)$ with an X-ray beam of wavelength $\lambda=1.560 \AA(\lambda=1.378 \AA)$. The X-ray scattering was measured as a function of $\mathrm{q}_{\text {radial }}=\mathrm{q}_{r}=(4 \pi / \lambda) \sin (2 \theta / 2)$, which is strain-sensitive, and also of $\mathrm{q}_{\text {angular }}=\mathrm{q}_{a}=(4 \pi / \lambda)$ $\sin (2 \theta / 2) \sin (2 \theta / 2-\omega)$, which is size sensitive, integrating $\mathrm{q}_{z}$ $=(2 \pi / \lambda)\left[\sin \left(\alpha_{i}\right)+\sin \left(\alpha_{f}\right)\right]$ from $0.02 \AA^{-1}$ to $0.07 \AA^{-1}$ [2]. In grazing incidence geometry, the scattering is due to atomic planes perpendicular to the sample surface and, essentially, two types of scans can be done. A radial scan is performed coupling $\omega$ to $2 \theta$, spanning from InAs to GaAs Bragg peaks in reciprocal space. Thus, by Bragg's law $(\lambda=$ $2 \mathrm{~d} \sin (2 \theta / 2))$, this radial scan is sensitive to the strain of the sample. Angular scans are performed solely by $\omega\left(\mathrm{q}_{a}\right)$, with $2 \theta\left(\mathrm{q}_{r}\right)$ fixed. These scans are size sensitive, since the size of the region can be inferred from the width of the $\mathrm{q}_{a}$-scan profile [3]. Fig. 1(b) shows a scheme of grazing incidence geometry.

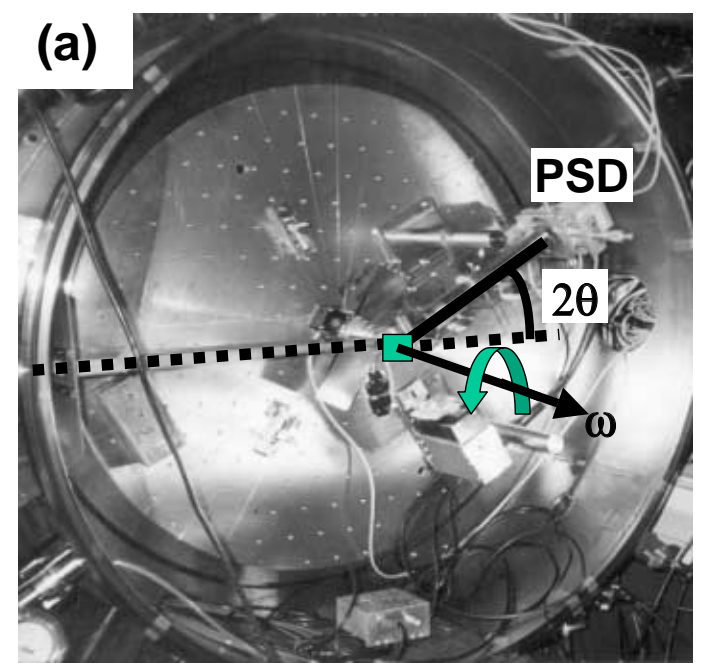

(b)

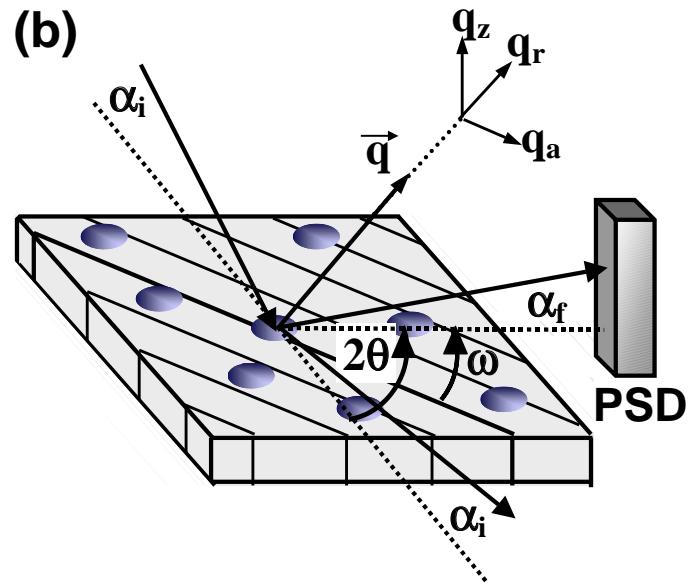

Figure 1. (a) X-ray scattering setup at XD1 beamline (LNLS). (b) Grazing incidence diffraction geometry.

Figure 2 shows two radial scans along the $\left(\begin{array}{l}2 \\ 2\end{array} 0\right)$ direction taken from samples A and C. Both samples present a reasonable amount of scattered $\mathrm{X}$-ray intensity between the
InAs and GaAs bulk peak positions. This is a clear evidence of the existence of a lattice parameter gradient inside the dots from the bottom to the top of the islands [2]. Besides the contribution from coherent dots, sample $\mathrm{C}$ shows some extra diffracted intensity near the InAs bulk peak position. Considering that this sample contains almost twice the amount of InAs than sample A and that islands with this coverage have already crossed the coherence-incoherence transition [1], we infer that this sample contains relaxed QDs with the lattice parameter of bulk InAs (6.06 $)$.

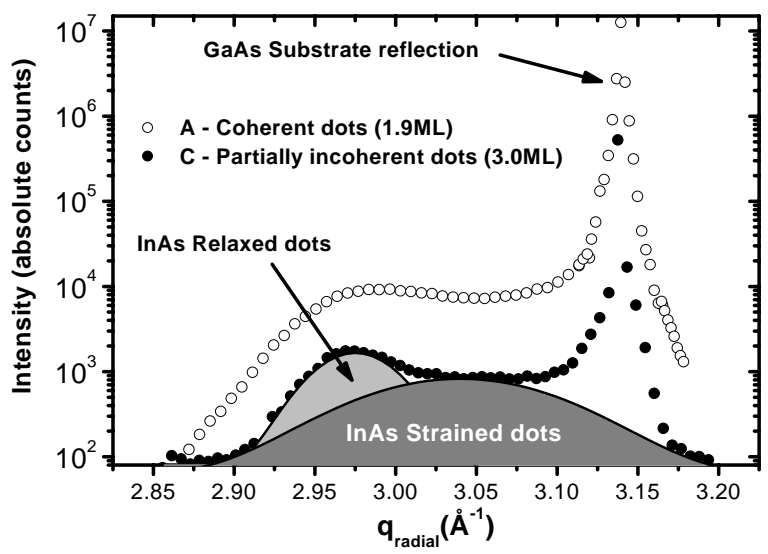

Figure 2. Radial scans for samples A and C. The two gaussians drawn for sample $\mathrm{C}$ to indicate the contributions of relaxed and strained islands.

In order to determine the relationship between island size and strain, angular $\mathrm{q}_{a}$-scans with fixed $\mathrm{q}_{r}$ were performed on samples A, B and C. Figs. 3.a and 3.b show these angular scans for samples $A$ and $C$, respectively, where $q_{a}$ corresponds to the [1-1 0$]$ direction. The scans start near the GaAs reciprocal lattice point $\left(\mathrm{q}_{r}=3.15 \AA^{-1}\right)$ and go up to the InAs position $\left(\mathrm{q}_{r}=2.94 \AA^{-1}\right)$. Since the full-width at half maximum of these diffraction profiles is inversely proportional to the size of the scattering object [3], a few statements can be drawn about the origin of the scattered intensity for each angular cut. In Fig. 3.a (sample A), as $\mathrm{q}_{r}$ decreases (cuts with larger lattice parameters) the central maximum broadens, indicating the decrease of the lateral dimension of scattering objects [4]. Hence, InAs dots are wide in regions where the lattice parameter is close to GaAs (i.e., the bottom of the islands) and constricted at the nearly relaxed top, as in ref. 2. The central maximum in sample C (Fig. 3.b) shows a somewhat different behavior than in sample A. From $\mathrm{q}_{r}=3.119 \AA^{-1}$ until $\mathrm{q}_{r}=2.994 \AA^{-1}$, the angular scans show the same broadening trend as in sample A. However, close to the InAs bulk peak position, the central maximum becomes sharp again, indicating the existence of larger relaxed islands [3]. 


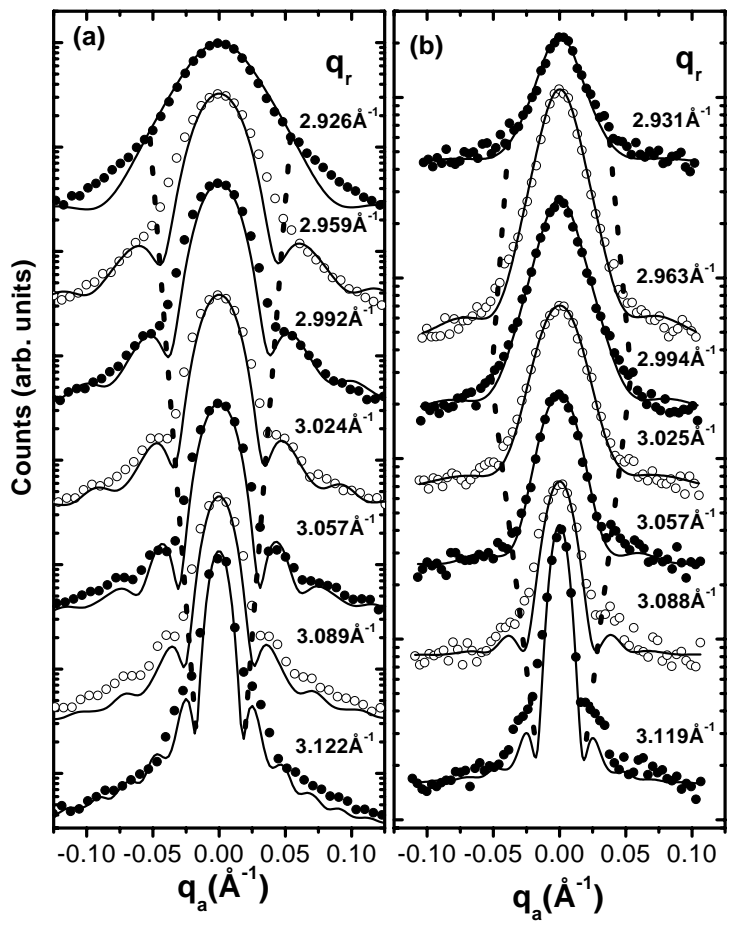

Figure 3. Angular scans at different radial $\left(\mathrm{q}_{r}\right)$ positions for (a) sample A and (b) sample. Solid lines are fits according to eq.1. Dashed lines were drawn to indicate the approximate width of each angular scan.

Based on AFM measurements of all samples, the islands have a pyramidal shape with a square section of side length $\mathrm{L}$ along the [1-10] direction and a varying local lateral lattice parameter. In this case, the scattered intensity of an angular $\mathrm{q}_{a}$-scan for a fixed $\mathrm{q}_{r}$ and $\mathrm{q}_{z}$ can be calculated using [3]

$$
I\left(q_{a}, q_{r}, q_{z}\right) \propto\left|\sum_{j=1}^{M} \frac{e^{i L_{j} q_{a}}-1}{q_{a}} \cdot \frac{e^{i N_{j} d_{j} q_{r}}-1}{e^{i d_{j} q_{r}}-1} e^{i q_{z} h_{j}}\right|^{2}
$$

where $\mathrm{M}$ is the number of square sections parallel to the surface, $L_{j}, d_{j}$ and $h_{j}$ are the length, the lattice parameter and the height of square section $\mathrm{j}$, respectively. $N_{j} \equiv L_{j} / d_{j}$ is the number of atomic lines at plane $\mathrm{j}$. The solid lines in Figs. 3 (a) and (b) are least-square fits done using eq.1, which were performed adjusting the square side length $\mathrm{L}$ for every fixed $\mathrm{q}_{r}$ (corresponding to a small region of the island of lattice parameter $\left.d=2 \pi / q_{r}\right)$.

A plot of the island side length $L$ as a function of lattice parameter $d$ is shown in Fig. 4. Sample A (coherent QDs) presents a linear decrease of the side length as a function of lattice parameter as reported before [2]. Samples B and $\mathrm{C}$, besides the contribution from coherent islands (close to GaAs value), also exhibit larger side lengths close to InAs value, which are, therefore, assigned to be relaxed islands (and correspond to an average value of the size of these islands, not a local lattice parameter). An unambiguous differentiation regarding size between coherent and incoherent islands was therefore obtained with this $\mathrm{x}$-ray technique.

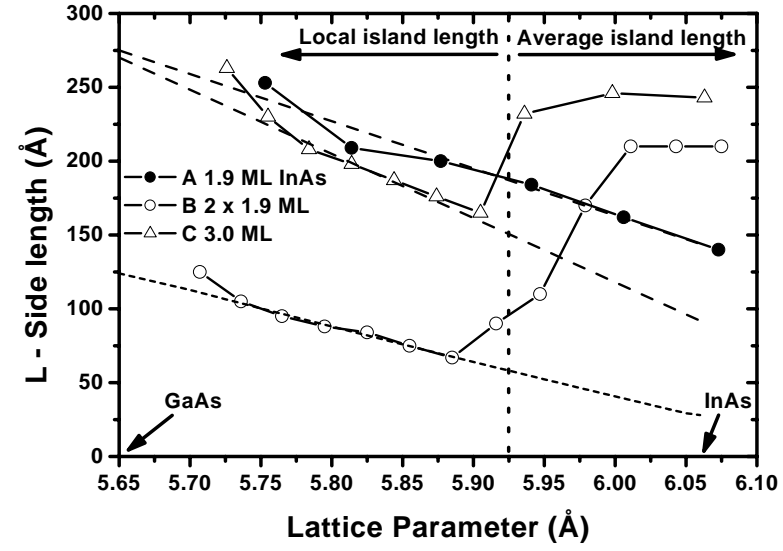

Figure 4. Islands side length as a function of lattice parameter for all samples. The straight lines are linear fits for coherent islands.

The linear fits done to points associated to coherent islands were in the format $\mathrm{L}(\Delta \mathrm{d})=\mathrm{L}_{0}-\mathrm{D} \Delta \mathrm{d}$, where $\mathrm{L}_{0}$ is the island bottom size (in $\AA$ ), $\mathrm{D}$ is a fitting parameter and $\Delta \mathrm{d}$ is the deviation from the GaAs lattice parameter $(\AA)$. We obtained $\mathrm{L}=280 \AA-130 \cdot \Delta \mathrm{d}, \mathrm{L}=120 \AA-100 \cdot \Delta \mathrm{d}$ and $\mathrm{L}=$ $270 \AA-170 \cdot \Delta \mathrm{d}$, for samples $\mathrm{A}, \mathrm{B}$ and $\mathrm{C}$, respectively.

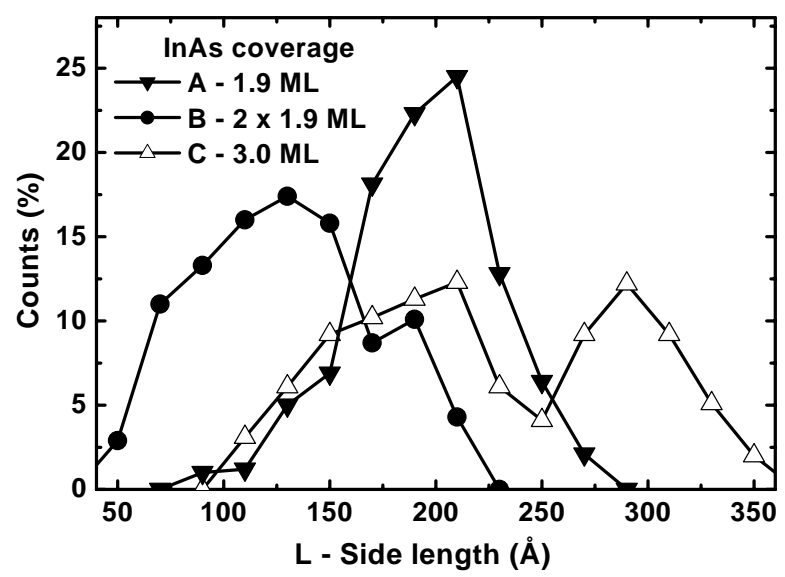

Figure 5. Size distribution graphs of all samples obtained from analysis of AFM images.

In order to confirm the ability of the x-ray technique to distinguish strained and relaxed islands, a direct comparison with AFM measurements was made. The graph in Fig. 5 shows the bottom side lengths as obtained from AFM images of all three samples. Sample A exhibits one type of QD, with a distribution centered at $\sim 200 \AA$ with a $\pm 60 \AA$ size dispersion. For sample $B$, the AFM data suggest a bimodal distribution of coherent QDs centered at $\sim 120 \AA$ and incoherent QDs centered at $\sim 200 \AA$. Although these AFM data are not conclusive regarding the bimodal nature of the distribution, they agree well with x-ray results, where the separation between strained and relaxed islands is quite clearer. A bimodal size distribution is also observed in sample C, centered at $\sim 190 \AA$ (strained QDs) and $\sim 300 \AA$ (relaxed QDs). Therefore, even though AFM and x-ray techniques are shown to portray good conformity, the $\mathrm{x}$-ray results are much more decisive in separating strained and relaxed islands in samples where the AFM analysis is inconclusive. 


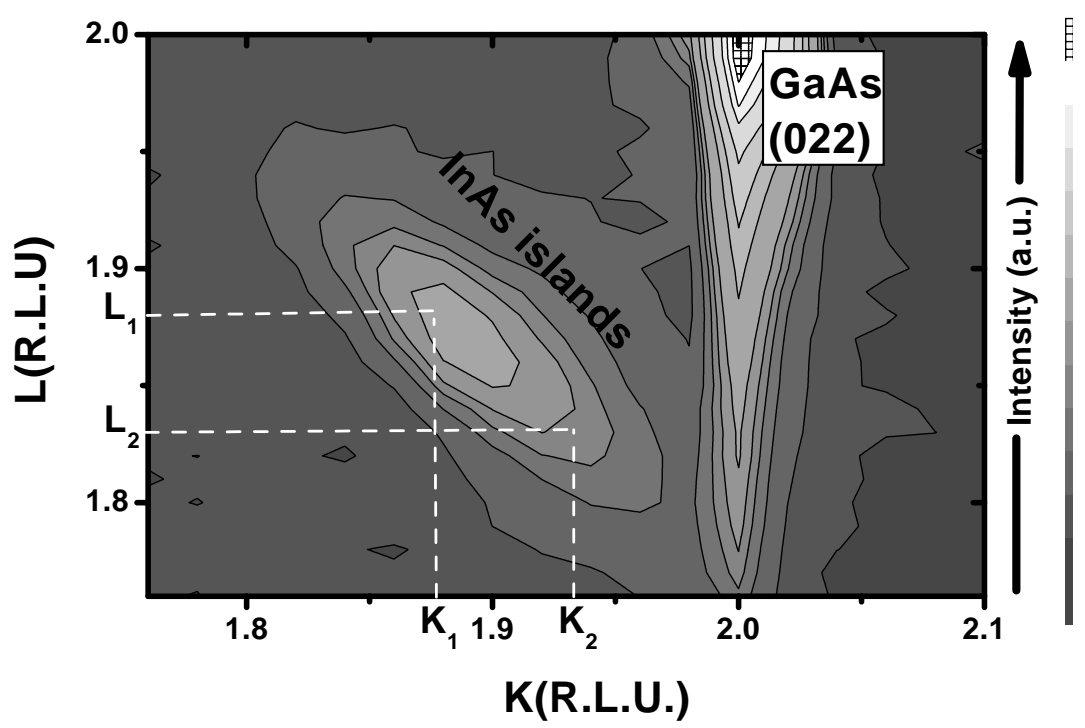

Figure 6. X-ray reciprocal space map of InAs/GaAs (001) islands near the (022) GaAs reflection. K and L reciprocal lattice units refer to the lattice of bulk GaAs. This scan allows us to associate horizontal and vertical lattice parameters, as represented by dashed lines for two pairs of values $\mathrm{K}_{1}, \mathrm{~L}_{1}$ and $\mathrm{K}_{2}, \mathrm{~L}_{2}$.

\section{Ga interdiffusion inside InAs dots}

X-ray reciprocal space mapping has already been used to study self-assembled islands, mainly using strain models to reproduce the intensity maps [4,5]. Here grazing incidence diffraction was used in combination with x-ray reciprocal space mapping to infer the three-dimensional strain and chemical status of InGaAs islands. With this information the tetragonal distortion of all unit cells inside the islands was quantified. The interdiffusion profile was then deduced from the elastic distortion of the unit cell determined by the Poisson ratio of the InGaAs alloy.

Following the GID measurements, $x$-ray reciprocal space mapping (in fixed incident angle mode) near the substrate GaAs (022) reflection was performed for sample C. The scattering measurements were performed at the XD2 beamline at the Brazilian Synchrotron Light Source (Laboratório Nacional de Luz Síncrotron), in Campinas, Brazil. This beamline is equipped with a double bounce sagitally focussed $\mathrm{Si}(111)$ monochromator and a standard 4-circle diffractometer. The incidence angle $\alpha_{i}$ was set to $0.1^{0}$ to maximize the islands/substrate signal ratio. The $\mathrm{x}$-ray mapping was measured by a point scintillation detector.

Figure 6 shows the reciprocal space intensity map of the $\mathrm{X}$-ray scattering stemming from sample $\mathrm{C}$. This rectangular reciprocal space map scan starts near the position of the relaxed (022) InAs reflection and goes up to the position of the (022) GaAs substrate peak, spanning from $\mathrm{K}=\mathrm{L}=$ 1.76 R.L.U. until $\mathrm{K}=2.10$ R.L.U and $\mathrm{L}=2.0$ R.L.U.. Two main contributions can be qualitatively identified. First, we clearly observe the crystal truncation rod along the $001 \mathrm{di}-$ rection crossing the (022) GaAs peak, parallel to the GaAs surface normal. We also observe the island scattering intensity distribution, which already shows the signature of the tetragonal distortion of the island lattice. The lateral (in- plane) compression of the InAs island lattice by the substrate leads to a vertical (out-of-plane) expansion. In reciprocal space this can be seen from the X-ray map, where for larger $\mathrm{K}$ the main intensity is seen for smaller $\mathrm{L}$.

In order to start a quantitative analysis of fig.6, a few assumptions were made. First, each portion of the islands at height $\mathrm{z}$ with respect to the substrate has a horizontal lattice parameter. This layer is constrained by a biaxial strain that creates a tetragonal distortion in the islands unit cells, changing the out-of-plane lattice parameter. Finally, there is a direct correlation between horizontal and vertical lattice parameters. These approximations are valid assuming that the island behaviour is similar to the bulk, meaning that it has the same elastic constants.

The measurement of Fig. 6 allowed us to correlate the inplane and out-of-plane lattice parameters. This association was obtained as follows: for each $\mathrm{L}$ scan for $\mathrm{K}$ fixed, an inplane lattice parameter $a_{/ /}=a_{G a A s} * 2 / \mathrm{K}$ was associated. The peak position of each L scan (determined from a gaussian fit) was associated to the out-of-plane lattice parameter $a_{\perp}=a_{\text {GaAs }} * 2 / L_{\text {peak }}$. In this way, pairs of values of $\left(a_{/ /}\right.$, $\left.a_{\perp}\right)$, obtained from $\left(K, L_{\text {peak }}\right)$ pairs, were obtained. The left plot of Fig. 7 shows values of $\left(a_{/ /}, a_{\perp}\right)$ related to regions of the island with these two lattice parameters.

Two factors can be responsible for changes in the unit cells volume inside the InAs islands: interdiffusion and elastic deformation (strain). As a starting point only interdiffusion was considered. By Vegard's law, a variation of lattice parameter of an alloy is linear with respect to its composition. The average lattice parameter for an $\mathrm{In}_{1-x} \mathrm{Ga}_{x}$ As alloy is given by $a_{\text {local }}=x \cdot a_{G a A s}+(1-x) \cdot a_{\text {InAs }}$, where $x$ is the $\mathrm{Ga}$ concentration and $a_{\text {local }}$ the local lattice parameter of the unit cell if it is not strained. The relationship between Ga concentration and the local lattice parameter $a_{\text {local }}$ is given by 


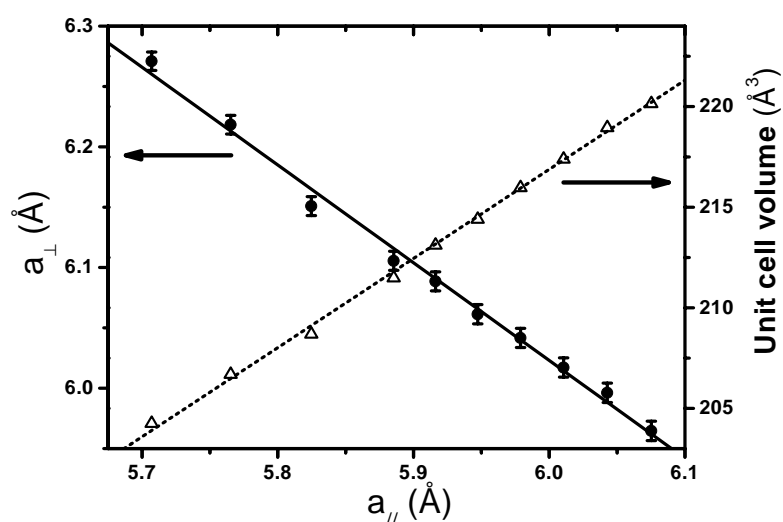

Figure 7. Left: correlation between in-plane and out-of-plane lattice parameters in InAs islands. Right: unit cell volume as a function of horizontal lattice parameter. The error bars are smaller than the symbol sizes.

$$
x=\frac{a_{\text {local }}-a_{\text {In } / A s}}{a_{\text {GaAs }}-a_{\text {InAs }}}
$$

One can calculate the unit cell volume from pairs of values $\left(a_{/ /}, a_{\perp}\right)$, considering that the unit cell is tetragonal, i.e., $V=a_{/ /}^{2} a_{\perp}$. The right plot of Fig. 7 shows the unit cell volume as a function of in-plane lattice parameter. This result shows that the unit cell volume diminishes as the inplane lattice parameter decreases. This is a first indication of the presence of $\mathrm{Ga}$ inside the islands. However, one must consider the effect of strain imposed by the substrate.

A more realistic approximation of the behaviour of this system can be obtained using the elastic properties of InAs and GaAs. The in-plane and out of plane strain components are defined as:

$$
\varepsilon_{/ /}=2 \frac{a_{/ /}-a_{\text {local }}}{a_{/ /}+a_{\text {local }}} \text { and } \varepsilon_{\perp}=2 \frac{a_{\perp}-a_{\text {local }}}{a_{\perp}+a_{\text {local }}}
$$

The relationship between vertical and horizontal strain components depends on the type of compression (axial or biaxial) the material is subjected to.

For a biaxial compression the strain components are related by [6]:

$$
\varepsilon_{\perp}=\frac{-2 \nu}{1 \nu} \epsilon_{/ /} .
$$

Inserting $\varepsilon_{\perp}$ and $\varepsilon_{/ /}$(equation 4) into eq. 5, a local can be found solving the equation:

$$
\left(a_{\text {local }}\right)^{2}(1+\nu)+a_{\text {local }}(1-3 \nu)\left(a_{\perp}-a_{/ /}\right)+a_{\perp} a_{\text {local }}(1+\nu)=0 .
$$

This quadratic equation has the following solutions

$$
a_{\text {local }}=\frac{(3 \nu-1)\left(a_{\perp}-a_{/ /}\right) \pm \sqrt{(1-3 \nu)^{2}\left(a_{\perp}-a_{/ /}\right)^{2}+4(1+\nu)^{2} a_{\perp} a_{/ /}}}{-2(1+\nu)}
$$

where one of the solutions leads to a negative lattice parameter. From the positive value of $a_{\text {local }}$ and eq. 2, the $\mathrm{Ga}$ concentration could be estimated.

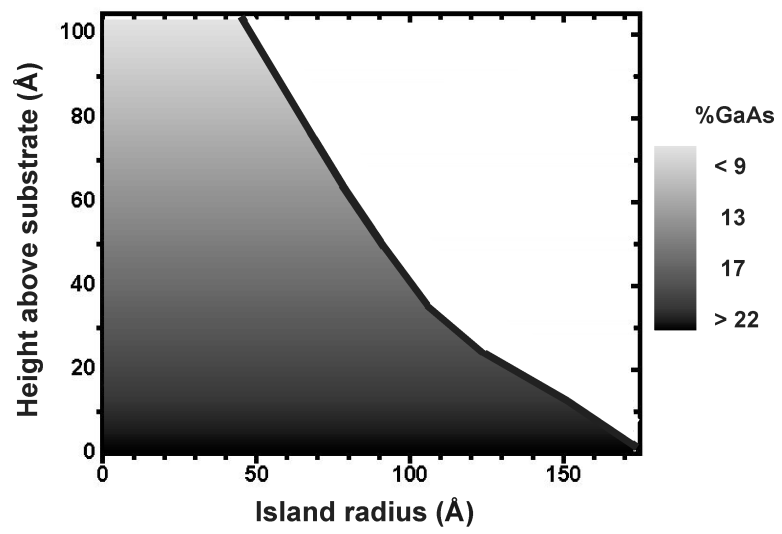

Figure 8. Ga content map in InAs:GaAs(001) islands determined using the method described in the text.

The InAs and GaAs Poisson ratios are 0.35 and 0.31 , respectively. A self-consistent procedure to calculate $a_{\text {local }}$ (eq. 7) was adopted: first the InAs Poisson ratio was used to obtain an initial estimate for the Ga concentration. A corrected value for the Poisson ratio was obtained from Vegard's law and the Ga concentration:

$$
\nu_{\text {alloy }}=x \cdot \nu_{\text {GaAs }}+(1-x) \cdot \nu_{\text {InAs }} .
$$

This new Poisson ratio was taken again to eq. 7 and new values for $a_{\text {local }}$ and $x$ were found. The result of this calculation can be seen in Fig. 8 .

The interpretation of the plot of Fig. 8 is straightforward. For regions close to substrate, where the horizontal lattice parameter is near GaAs value the Ga concentration is higher $(\sim 25 \%)$. Closer to the island top, the presence of $\mathrm{Ga}$ is smaller. This result is probably due to kinetic limited Ga interdiffusion during the deposition of InAs.

\section{Conclusion}

In conclusion, $\mathrm{x}$-ray scattering was employed to directly observe the coexistence of strained and relaxed nanostructures in partially coherent InAs quantum dot samples. It was 
shown that the $\mathrm{x}$-ray scattering technique clearly separates these two island types even when coherent and incoherent QDs have about the same size. We have also used reciprocal space mapping under grazing incidence geometry to study the local tetragonal distortion of InAs islands grown on $\mathrm{GaAs}(001)$. For the case of sample $\mathrm{C}$ we observed a variation of $\mathrm{Ga}$ content from $25 \%$ (base of the island) up to $8 \%$ (top of the islands). This method is in principle capable of determining the complete three-dimensional strain and chemical status of any island-substrate system.

\section{Acknowledgement}

The authors acknowledge support from PRONEX, FAPEMIG, CNPq and LNLS (ABTLus).

\section{References}

[1] D. Leonard, K. Pond, P.M. Petroff, Phys. Rev. B 50, 11687 (1994).

[2] I. Kegel, T. H. Metzger, J. Peisl, A. Lorke, J. M. Garcia, P. M. Petroff, Europhys. Lett. 45, 222 (1999); I. Kegel, T. H. Metzger, A. Lorke, J. Peisl, J. Stangl, G. Bauer, P. M. Petroff, Phys. Rev. B, 63, 35318 (2001)

[3] J.M. Cowley, Diffraction Physics, Ed. North-Holland, (1981).

[4] K. Zhang, Ch. Heyn, W. Hansen, Th Schmidt, and J. Falta, Appl. Phys. Lett. 77, 1295 (2000).

[5] J. Stangl, A. Daniel, V. Holy, T. Roch, G. Bauer, I. Kegel, T. H. Metzger, O. G. Schmidt, and K. Eberl, Appl. Phys. Lett. 79, 1474 (2001).

[6] See, e.g., J.Y. Tsao, Materials Fundamentals of Molecular Beam Epitaxy (Academic Press, London, 1993). 